East African Medical Journal Vol. 78 No. 6 June 2001

TRACHEA STABILISATION WITH AUTOLOGOUS COSTAL CARTILAGE IN ACOUIRED TRACHEOMALACIA: REPORT OF TWO CASES

K. Frimpong-Boateng MBChB, MD, FWACS, Head, Department of Surgery and Director, National Cardiothoracic Centre and E. Aniteye, MBChB, DA (UK), FRCA (UK),

Consultant Anaesthetist, National Cardiothoracic Centre, University of Ghana Medical School, P.O. Box 4236, Accra, Ghana.

\title{
TRACHEA STABILISATION WITH AUTOLOGOUS COSTAL CARTILAGE IN ACQUIRED TRACHEOMALACIA: REPORT OF TWO CASES
}

\section{K. FRIMPONG-BOATENG and E. ANITEYE}

\begin{abstract}
SUMMARY
Post-operative tracheomalacia is a life threatening condition whose management is challenging. Surgical procedures which have been suggested in the literature to manage the condition include tracheostomy, staged thyroid reductions and the use of artificial stents either within the lumen of the tracheobronchial tree or as external support. We report the successful management of two patients using autologous costal cartilage to support the tracheal wall.
\end{abstract}

\section{INTRODUCTION}

Tracheomalacia refers to weakness of the trachea wails and the supporting cartilage with easy collapsibility. It may present as a primary lesion especially in children where it is associated with a deficiency of cartilage in the tracheobronchial tree(1-3). In most cases, however, it is seen as a secondary lesion such as following pressure necrosis of cartilage due to intubation, radiotherapy(4), vascular abnormality(5) or relapsing polychondritis(6). An important cause of tracheomalacia is neglected goitre especially those with intrathoracic extension and after thyroidectomy(7-9). These goitres may also cause compression symptoms such as dysphagia, superior vena cáva compression, and Horner's syndrome. Postthyriodectomy tracheomalacia is a life threatening condition whose management may be difficult as evidenced by the many surgical procedures that have been suggested for it(9). We publish our experience with the use of autologous costal cartilage to stabilise the trachea wall after thyroidectomy. Our method is relatively simple, cost effective and has few side effects.

Surgical procedure of trachea stabilisation with autologous costal cartilage (Figure 1): With the patient in the supine position a $5-10 \mathrm{~cm}$ incision is made over the 5 th costal cartilage. The cartilage is dissected and removed. Care is taken not to open the pleural cavity. A chest tube is inserted if the pleura is inadvertently opened. The cartilage is split into two halves on a wooden or plastic board using a sharp scarpel blade. An adequate length of each half of the cartilage is placed on either side of the whole length of the trachea. Each cartilage is secured into place with a series of three or four number 0 double armed vicryl sutures which go through the external layers of the wall of the trachea. The series of sutures are tied over the cartilage. The two arms of each suture pair are passed through the sternomastoid muscle from medial to the lateral border on either side of the trachea. The sutures are then tied on the lateral aspect of the sternomastoid muscle. The wound is closed after adequate drainage. The patient is extubated on the table.
Figure 1

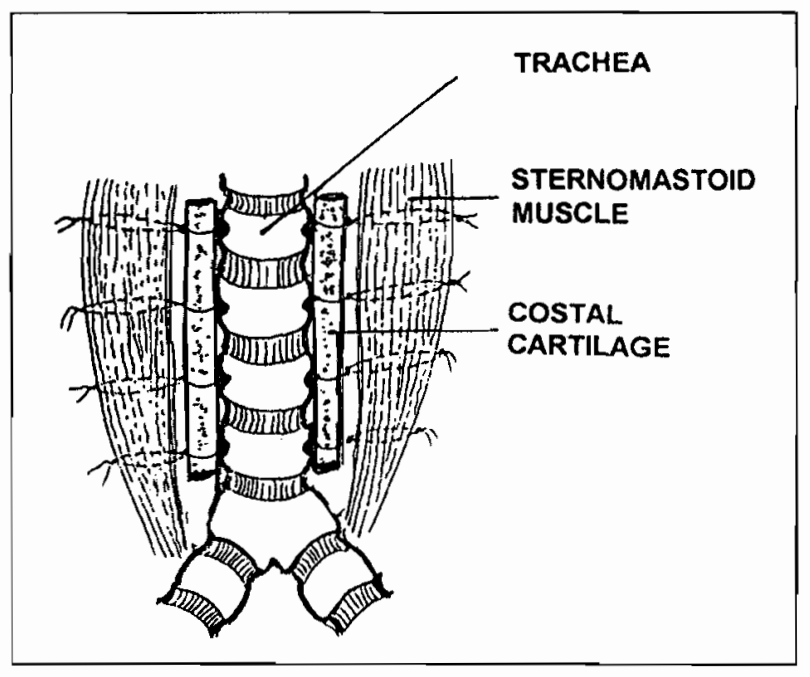

\section{CASE REPORT}

Case 1: A 67-year old Ghanaian male was admitted in February 1994 with a goitre he had for 22 years. For the last six years he experienced increasing dysphagia, stridor even at rest and progressive swelling of the face and hoarseness of voice. He had no chronic obstructive airway disease or emphysema. Thyroid function tests showed euthyroid function. $X$-rays and CT-scan of neck and thorax showed deviation and narrowing of the trachea. The narrowing was from the junction of the upper one- third and the middle one-third to about $2 \mathrm{~cm}$ above the carina. At surgery fibreoptic laryngoscopy was performed to aid a rather difficult intubation. A neck collar incision was made to expose a multinodular goitre. A total thyroidectomy was performed. The upper sternum was split to facilitate the excision of the intrathoracic extension of the goitre. During the dissection the wall of the trachea collapsed under the weight of the intra thoracic goitre. An assistant had to lift it till it was excised. An examination of the trachea showed that its wall was thinned out and the supporting cartilage rings were atrophied and weak. The anaesthetist was told to withdraw the endotracheal tube. In the process the wall of the narrowed segment of the trachea, about $6 \mathrm{~cm}$ in length, collapsed. The trachea was stabilized using autologous costal cartilage. The patient was extubated on the 
table. He was discharged after 10 days. During the first three post-operative follow-ups at four weeks, three months and six months respectively antero-posterior and lateral conventional $\mathrm{x}$ rays of the neck and chest as well as CT-scan of the neck and chest showed that the position of the trachea as well as the size and shape of its lumen were normal. Subsequently conventional $\mathrm{X}$-rays of the neck and chest were done at half yearly follow-ups. There have been no abnormalities and no complaints after six years follow up.

Case 2: A 55-year old Ghanaian female who had a large multinodular euthyroid goitre with stridor and increasing dysphagia was admitted in August 1991 and scheduled for thyroidectomy under the care of a general surgeon. She did not have any evidence of chronic obstructive airway disease. Preoperative evaluation showed intrathoracic extension with deviation of the narrowed trachea to the right. At surgery the intubation was normal. The goitre and its intrathoracic extension could be excised through a collar incision in the neck. The patient went into stridor after extubation. The vocal cords were normal on laryngoscopy. The cardiothoracic team was called to the operating room. The wound was re-opened and the trachea examined. Its wall. from the middle one-third to about $2.5 \mathrm{~cm}$ above the carina, a distance of about $5.5 \mathrm{~cm}$ was weak. The caritilage rings were thinned out. The tracheal wall was supported using autologous costal cartilage. The patient was extubated successfully. She was discharged home after 12 days. Postoperative follow-up studies of the neck and chest with conventional $\mathrm{x}$-rays and $\mathrm{C} T$-scan as case one have not shown any abnormalities. The patient has had no respiratory symptoms after nine years follow up.

\section{DISCUSSION}

Tracheomalacia following thyroidectomy can be a life-threatening postoperative complication. It may result in airway obstruction and death. Several methods have been described by other authors to deal with this problem. These include tracheostomy, staged tracheoplasty, staged thyroid reductions, insertion of metallic stents and extrinsic tracheal neorings using surgical wire or vascular prosthesiss(11). All these methods have potential serious drawbacks. Staged tracheoplasty and staged thyroid reduction involve more than one surgical operations with attendant morbidity. Tracheostomy has its inherent complications such as infection which can worsen the original condition, bleeding from erosion into surrounding tissues, displacement of tracheostomy tube and fracture of the tube which may lead to airway obstruction. Problems associated with the use of artificial material for the support of the trachea may include the risk of infection, perforation of the trachea and extrusion of the material, haemorrhage, fistula formation and stent migration(10,11). Tracheal resection, especially resection of relatively long segments as encountered in these two patients, is not always feasible in elderly patients with more rigid trachea. There is also the risk of re-stenosis. The use of autologous costal cartilage involves only one surgical session.
The risk of infection is not increased. The surgical procedure itself is relatively simple and cost effective and there is no foreign material to cause the problems mentioned above. The patient can be extubated soon after surgery and the long term result is excellent. Irrespective of the length of the collapsed segment of the trachea it is advisable not to limit the stabilisation to the unstable segment only but rather to apply the autologous costal cartilage to almost the whole length of the trachea in as much the same way as fractured bones are stabilised. Although there are no absolute contra-indications related to the surgical procedure there may be technical problems in patients who have had extensive neck dissection for other reasons or in patients with previous cervical oesophago-colonostomy. Moreover patients with previous chest wall irradiation may not be good candidates for removal of costal cartilages. A relative disadvantage of this technique is that the patient will have another scar on the chest wall. Surgically there is the remote possibility of injury to the carotid sheath and its contents and the phrenic nerve. The sternomastoid muscle is, however, easily identifiable and can be dissected from neighbouring structures with ease. No problems were encountered surgically. The method is so simple that one need not worry about a learning curve. In any case postoperative tracheomalacia is not a frequent occurrence and this surgical technique should be seen as an alternative for aclinical condition which is otherwise difficult to manage.

\section{REFERENCES}

1. Cogbill T. H., Moore F. A. and Accurso F. J. et al: Primary tracheomalacia. Amer. Thorac. Surg. 1983; 35:538.

2. Williams H., Campbell: Generalized bronchiectasis associated with deficiency of cartilage in the bronchial tree. Arch. Dis. Child $1960 ; 35: 182$

3. Santoli E., Di Biasi P. and Vanelli P. et al: Tracheal obstruction due to congenital tracheomalacia in a child. Case Report. Scand. J. Thorac. Cardiovasc. Surg. 1991; 25: 227.

4. Chetty K. G., Kadife F., Berry R. B. and Mahutte C. K. Acquired laryngomalacia as a cause of obstructive apnea. Chest 1994; 106: 1898 .

5. van Son J. A., Julsrud P. R. and Hagler D. J. et al: Surgical treatment of vascular rings: The Mayo Clinic Experience. Mayo Clin. Proc. $1993 ; 68$ : 1056

6. Feist J.H., Johnson T. H. and Wilson R. J. Acquired tracheomalacia, etiology and differential diagnosis Chest 1975; 68:340.

7. Newman E. and Shaha A. R. Substernal goiter. J. Surg. Oncol. 1995; 60:207.

8. Krishnan H. and May R.E: An unusual cause for respiratory difficulty after thyroidectomy. Brit. J. Clin. Pract. 1993; 47:47.

9. Geelhaed G.W. Tracheomalacia from compressing goitre, management after thyroidectomy. Surgery 1988; 104:1100-1108.

10. George P.J.M., Irving J.D. and Khanghani A et al. Role of the Gianturco expandable metal stent in the management of tracheobronchial obstruction. Cardiovasc. Intervent Radiol 1992; 15:375-381

11. Kim W., Lee Y.C. and Rhee Y.K. Obstruction after self-expanding metallic stents in tuberculous bronchial stenosis. Kor. J. Intern. Med. 1995; 10:64-67. 